\title{
Erratum
}

\section{2-Matrix versus Complex Matrix Model, Integrals over the Unitary Group as Triangular Integrals}

\section{B. Eynard ${ }^{1}$, A. Prats Ferrer ${ }^{2}$}

${ }^{1}$ Service de Physique Théorique de Saday, CEA/DSM/SPhT-CNRS/SPM/URA 2306,

91191 Gif-sur-Yuette Cedex, France. E-mail: Bertrand.Eynard@cea.fr

2 Departamenta d'Estructura i constituents de la Matèria, Universitad Barcelona, Av. Diagonal 647, 08028 Barcelona, Spain. E-mail: prats@ecm.ub.es

Received: 5 September 2006 / Accepted: 11 September 2007

Published online: 15 November 2007 - (C) Springer-Verlag 2007

Commun. Math. Phys. 264, 115-144 (2006)

In the above article Theorem 3.4 contains an error which is easily resolved through a slight reformulation of the statement, including in it part of Theorem 4.1. We keep the conventions and definitions given in the paper.

\section{Original Version}

This is Theorem 3.4 as it appears in the published article.

Theorem 3.4. For any polynomial invariant function $F(A, B)$, one has:

$$
\begin{aligned}
& \frac{\tilde{J}_{n}^{2}}{n !^{2} Z_{H}\left(n, \gamma, \alpha_{1}, \alpha_{2}\right)} \int_{D_{n}(\mathrm{R}) \times D_{n}(R)} d X d Y \mathrm{e}^{-\frac{\alpha_{1}}{2} \operatorname{Tr} X^{2}} \mathrm{e}^{-\frac{\alpha_{2}}{2} \operatorname{Tr} Y^{2}} \tilde{W}_{F}(X, Y) \\
& =\frac{J_{n}}{n ! Z_{C}\left(n, \gamma, \alpha_{1}, \alpha_{2}\right)} \int_{D_{n}(\mathrm{C})} d X \mathrm{e}^{-\frac{\alpha_{1}}{2} \operatorname{Tr} X^{2}} \mathrm{e}^{-\frac{\alpha_{2}}{2} \operatorname{Tr} \bar{X}^{2}} W_{F}(X, \bar{X}) .
\end{aligned}
$$

Proof. Start from Theorem 3.3, diagonalize $M_{1}$ and $M_{2}$ on the hermitian side, and jordanize $Z$ on the complex side,

$$
\begin{aligned}
\langle F\rangle_{H} & =\frac{\tilde{J}_{n}^{2}}{n !^{2} Z_{H}\left(n, \gamma, \alpha_{1}, \alpha_{2}\right)} \int_{D_{n}(\mathrm{R}) \times D_{n}(R)} d X d Y \mathrm{e}^{-\frac{\alpha_{1}}{2} \operatorname{Tr} X^{2}} \mathrm{e}^{-\frac{\alpha_{2}}{2} \operatorname{Tr} Y^{2}} \tilde{W}_{F}(X, Y) \\
=\langle F\rangle_{C} & =\frac{J_{n}}{n ! Z_{C}\left(n, \gamma, \alpha_{1}, \alpha_{2}\right)} \int_{D_{n}(\mathrm{C})} d X \mathrm{e}^{-\frac{\alpha_{1}}{2} \operatorname{Tr} X^{2}} \mathrm{e}^{-\frac{\alpha_{2}}{2} \operatorname{Tr} \bar{X}^{2}} \mathrm{e}^{-\gamma \operatorname{Tr} X \bar{X}} \omega_{F}(X, \bar{X})
\end{aligned}
$$




$$
\begin{aligned}
& =\frac{J_{n}}{n ! Z_{C}\left(n, \gamma, \alpha_{1}, \alpha_{2}\right)} \int_{D_{n}(\mathrm{C})} d X \mathrm{e}^{-\frac{\alpha_{1}}{2} \operatorname{Tr} X^{2}} \mathrm{e}^{-\frac{\alpha_{2}}{2} \operatorname{Tr} \bar{X}^{2}} \mathrm{e}^{-\gamma \operatorname{Tr} X_{\sigma} \bar{X}_{\tau}} \omega_{F}\left(X_{\sigma}, \bar{X}_{\tau}\right) \\
& =\frac{J_{n}}{n ! Z_{C}\left(n, \gamma, \alpha_{1}, \alpha_{2}\right)} \int_{D_{n}(\mathrm{C})} d X \mathrm{e}^{-\frac{\alpha_{1}}{2} \operatorname{Tr} X^{2}} \mathrm{e}^{-\frac{\alpha_{2}}{2} \operatorname{Tr} \bar{X}^{2}} W_{F}\left(X_{\sigma}, \bar{X}_{\tau}\right) .
\end{aligned}
$$

The equality in the first line is obtained by diagonalizing $M_{1}$ and $M_{2}$ (with Jacobian given in Eq.2-17), the equality in the second line is obtained by Jordanizing $Z$ (with Jacobian given in Eq.2-19), the equality between the second and third line holds for any pair of permutations $\sigma$ and $\tau$ (it can be proven with Lemma A.1 given in the Appendix), and the equality of the last line comes from the definition of $W_{F}$.

The main mistake appears from second to third line, since at this point it is false that permuting the two matrices $X$ and $\bar{X}$ to $X_{\sigma}$ and $\bar{X}_{\tau}$ independently does not change the integral. However, a small rearrangement of Theorems 3.4 and 4.1 yields the desired result.

\section{Corrected Version}

To fix the mistake, we reformulate Theorem 3.4 as follows:

Theorem 3.4. For any polynomial invariant function $F(A, B)$, one has:

$$
\begin{gathered}
\frac{\tilde{J}_{n}^{2}}{n !^{2} Z_{H}\left(n, \gamma, \alpha_{1}, \alpha_{2}\right)} \int_{D_{n}(\mathrm{R}) \times D_{n}(\mathrm{R})} d X d Y \mathrm{e}^{-\frac{\alpha_{1}}{2} \operatorname{Tr} X^{2}} \mathrm{e}^{-\frac{\alpha_{2}}{2} \operatorname{Tr} Y^{2}} \tilde{W}_{F}(X, Y) \\
=\frac{J_{n}}{n ! Z_{C}\left(n, \gamma, \alpha_{1}, \alpha_{2}\right)} \int_{D_{n}(\mathrm{R}) \times D_{n}(\mathrm{R})} d X d Y \mathrm{e}^{-\frac{\alpha_{1}}{2} \operatorname{Tr} X^{2}} \mathrm{e}^{-\frac{\alpha_{2}}{2} \operatorname{Tr} Y^{2}} W_{F}(X, Y) .
\end{gathered}
$$

Proof.

$$
\begin{aligned}
& \langle F\rangle_{H}=\frac{\tilde{J}_{n}^{2}}{n !^{2} Z_{H}\left(n, \gamma, \alpha_{1}, \alpha_{2}\right)} \int_{D_{n}(\mathrm{R}) \times D_{n}(\mathrm{R})} d X d Y \mathrm{e}^{-\frac{\alpha_{1}}{2} \operatorname{Tr} X^{2}} \mathrm{e}^{-\frac{\alpha_{2}}{2} \operatorname{Tr} Y^{2}} \tilde{W}_{F}(X, Y) \\
& =\langle F\rangle_{C}=\frac{J_{n}}{n ! Z_{C}\left(n, \gamma, \alpha_{1}, \alpha_{2}\right)} \int_{D_{n}(\mathrm{C})} d X \mathrm{e}^{-\frac{\alpha_{1}}{2} \operatorname{Tr} X^{2}} \mathrm{e}^{-\frac{\alpha_{2}}{2} \operatorname{Tr} \bar{X}^{2}} \mathrm{e}^{-\gamma \operatorname{Tr} X \bar{X}} \omega_{F}(X, \bar{X}) \\
& =\frac{J_{n}\left(\frac{\pi}{\sqrt{-\delta}}\right)^{n}}{n ! Z_{C}\left(n, \gamma, \alpha_{1}, \alpha_{2}\right)\left(\frac{2 \pi}{\sqrt{\delta}}\right)^{n}} \int_{D_{n}(\mathrm{R}) \times D_{n}(\mathrm{R})} d X d Y \mathrm{e}^{-\frac{\alpha_{1}}{2} \operatorname{Tr} X^{2}} \\
& \times \mathrm{e}^{-\frac{\alpha_{2}}{2} \operatorname{Tr} Y^{2}} \mathrm{e}^{-\gamma \operatorname{Tr} X Y} \omega_{F}(X, Y) \\
& =\frac{J_{n}\left(\frac{\pi}{\sqrt{-\delta}}\right)^{n}}{n ! Z_{C}\left(n, \gamma, \alpha_{1}, \alpha_{2}\right)\left(\frac{2 \pi}{\sqrt{\delta}}\right)^{n}} \frac{1}{n !^{2}} \\
& \times \sum_{\sigma, \tau} \int_{D_{n}(\mathrm{R}) \times D_{n}(\mathrm{R})} d X d Y \mathrm{e}^{-\frac{\alpha_{1}}{2} \operatorname{Tr} X^{2}} \mathrm{e}^{-\frac{\alpha_{2}}{2} \operatorname{Tr} Y^{2}} \mathrm{e}^{-\gamma \operatorname{Tr} X_{\sigma} Y_{\tau}} \omega_{F}\left(X_{\sigma}, Y_{\tau}\right) \\
& =\frac{J_{n}\left(\frac{\pi}{\sqrt{-\delta}}\right)^{n}}{n ! Z_{C}\left(n, \gamma, \alpha_{1}, \alpha_{2}\right)\left(\frac{2 \pi}{\sqrt{\delta}}\right)^{n}} \frac{1}{n !^{2}} \\
& \times \sum_{\sigma, \tau} \int_{D_{n}(\mathrm{R}) \times D_{n}(\mathrm{R})} d X d Y \mathrm{e}^{-\frac{\alpha_{1}}{2} \operatorname{Tr} X^{2}} \mathrm{e}^{-\frac{\alpha_{2}}{2} \operatorname{Tr} Y^{2}} W_{F}(X, Y) .
\end{aligned}
$$


The equality in the first line is obtained by diagonalizing $M_{1}$ and $M_{2}$ (with Jacobian given in Eq.2-17), the equality in the second line is obtained by Jordanizing $Z$ (with Jacobian given in Eq.2-19), the equality between the second and third line follows from Lemma A.1 given in the Appendix, the equality between the third and fourth line can be done thanks to the symmetry of the measure. It is convenient to do so in order to compare integrands later, since the integrand in the first line is symmetric by the action of the unitary integral inside $\tilde{W}_{F}(X, Y)$. Finally the equality between the fourth and fifth line comes from the definition of $W_{F}$.

Additional minor modification. Due to the inclusion of part of Theorem 4.1 in the corrected Theorem 3.4, we must rewrite the proof of the former in the following terms

Theorem 4.1. For any invariant function $F(A, B)$ one has:

$$
\begin{aligned}
& \int_{U(n)} d U F\left(X, U Y U^{\dagger}\right) \mathrm{e}^{-\gamma \operatorname{Tr} X U Y U^{\dagger}} \\
& =\frac{c_{n}}{n !} \frac{\sum_{\sigma} \sum_{\tau}(-1)^{\sigma}(-1)^{\tau} \mathrm{e}^{-\gamma \operatorname{Tr} X_{\sigma} Y_{\tau}} \int_{T(n)} F\left(X_{\sigma}+T, Y_{\tau}+T^{\dagger}\right) \mathrm{e}^{-\gamma \operatorname{Tr} T T^{\dagger}} d T}{\Delta(X) \Delta(Y)},
\end{aligned}
$$

where

$$
c_{n}=\frac{\prod_{k=0}^{n-1} k !}{(-2 \pi)^{\frac{n(n-1)}{2}}},
$$

i.e.

$$
\tilde{W}_{F}(X, Y)=n ! c_{n} W_{F}(X, Y) .
$$

Proof. From Theorem 3.4 we have

$$
0=\int_{D_{n}(\mathrm{R}) \times D_{n}(\mathrm{R})} d X d Y \mathrm{e}^{-\frac{\alpha_{1}}{2} \operatorname{Tr} X^{2}} \mathrm{e}^{-\frac{\alpha_{2}}{2} \operatorname{Tr} Y^{2}}\left(n ! c_{n} W_{F}(X, Y)-\tilde{W}_{F}(X, Y)\right) .
$$

Notice that if $f(A)$ and $g(B)$ are invariant functions, i.e. $f\left(U A U^{-1}\right)=f(A)$ for all $A$ and $U$ (resp. $g\left(U B U^{-1}\right)=g(B)$ for all $B$ and $U$ ), one has:

$$
\begin{aligned}
& W_{f(X) g(Y) F(X, Y)}(X, Y)=f(X) g(Y) W_{F}(X, Y), \\
& \tilde{W}_{f(X) g(Y) F(X, Y)}(X, Y)=f(X) g(Y) \tilde{W}_{F}(X, Y) .
\end{aligned}
$$

Thus, for any symmetric polynomials $f(x 1, \ldots, x n)$ and $g(y 1, \ldots, y n)$, one has:

$$
\begin{aligned}
0= & \int_{D_{n}(\mathrm{R}) \times D_{n}(\mathrm{R})} d X d Y \mathrm{e}^{-\frac{\alpha_{1}}{2} \operatorname{Tr} X^{2}} \mathrm{e}^{-\frac{\alpha_{2}}{2} \operatorname{Tr} Y^{2}} f(X) g(Y) \\
& \times\left(n ! c_{n} W_{F}(X, Y)-\tilde{W}_{F}(X, Y)\right) .
\end{aligned}
$$

Notice that $\mathrm{e}^{-\frac{\alpha_{1}}{2} \operatorname{Tr} X^{2}} \mathrm{e}^{-\frac{\alpha_{2}}{2} \operatorname{Tr} Y^{2}}\left(n ! c_{n} W_{F}(X, Y)-\tilde{W}_{F}(X, Y)\right)$ is a continuous function quickly decreasing at $\infty$ in all variables, and it is symmetric in the $x$ 's and in the $y$ 's. Using the Stone-Weierstrass Theorem (polynomials are dense in the space of continuous functions), one gets that $\left(n ! c_{n} W_{F}(X, Y)-\tilde{W}_{F}(X, Y)\right)=0$. 